\title{
Blind Resolution of Lifetime Components in Individual Pixels of Fluorescence Lifetime Images Using the Phasor Approach
}

\author{
Alexander Vallmitjana1, \# , Belén Torrado ${ }^{1, \#}$, Alexander Dvornikov${ }^{1}$, \\ Suman Ranjit ${ }^{2,}{ }^{*}$ and Enrico Gratton ${ }^{1, *}$ \\ ${ }^{1}$ Laboratory for Fluorescence Dynamics, Biomedical Engineering, University of California, Irvine, California. \\ ${ }^{2}$ Department of Biochemistry and Molecular \& Cellular Biology, Georgetown University, Washington D.C. \\ \#Contributed equally. \\ *egratton22@gmail.com, suman.ranjit@georgetown.edu corresponding authors.
}

\section{Supplemental material}

\section{Formalism}

In the phasor approach the fluorescence lifetime decay curve in every pixel $I(t)$ is transformed using the integral expressions that give the coordinates on the phasor plot for that pixel at a particular laser angular frequency $\omega=2 \pi f$ and harmonic $n$ for the transform:

$$
\begin{aligned}
S & =\frac{\int_{0}^{T} I(t) \sin (n \omega t) d t}{\int_{0}^{T} I(t) d t} \\
G & =\frac{\int_{0}^{T} I(t) \cos (n \omega t) d t}{\int_{0}^{T} I(t) d t}
\end{aligned}
$$

The harmonic number $n$, representing the integer number of whole cycles the trigonometric function has in the repetition period. $T$ is the repetition frequency of the laser. Each value of the harmonic provides a different pair of coordinates $(S$ and $G$ ) and the function to be transformed is the mathematical expression for a decaying exponential.

By plugging $(t)=A e^{\frac{-t}{\tau}}$, that is forcing that the intensity decay curves are pure exponential, we can solve the integrals in Eqs 1 and 2 to obtain the expressions:

$$
s=\frac{n \omega \tau}{1+(n \omega \tau)^{2}}
$$




$$
g=\frac{1}{1+(n \omega \tau)^{2}}
$$

Which can be combined to remove the dependency on the frequency and find the equation of the points on the plane where the pure decays lie; namely the universal semicircle:

$$
s_{i}^{2}=g_{i}-g_{i}^{2}
$$

When the intensity decay curve is some combination of exponential curves; $I(t)=\sum_{1}^{N} A_{i} e^{\frac{-t}{\tau}}$, the resulting coordinates of the phasor transform are the sum of the phasor transforms of the pure components, therefore the weighted sum of positions on the universal circle:

$$
\begin{aligned}
& \sum_{1}^{N} f_{i} g_{i}^{(h)}=G^{(h)} \\
& \sum_{1}^{N} f_{i} s_{i}^{(h)}=S^{(h)}
\end{aligned}
$$

Upper case $\left(S^{(h)}, G^{(h)}\right)$ are the coordinates of our data points in each harmonic, lower case $\left(s_{i}^{(h)}, g_{i}^{(h)}\right)$ are the unknown coordinates of the pure components and $f_{i}$ their respective photon fractions. Upper indices in parenthesis $(h)$ correspond to the harmonic number. Needless to say, the fractions have to add up to unity:

$$
\sum_{1}^{N} f_{i}=1
$$

One last constraint that can be imposed is the relationship between the harmonics of the pure exponential decays that lie on the universal circle. From the solution of the integral expressions on the phasor plot of the phasor transform (Eq 3 and Eq. 4), they can be rearranged to express a particular harmonic $m$ in terms of another harmonic $n$ :

$$
g^{(n)}=\frac{m^{2} g^{(m)}}{n^{2}+\left(m^{2}-n^{2}\right) g^{(m)}}
$$

Again, note that here the superscripts in $\left(g^{(m)}, g^{(n)}\right)$ refer to the $n^{\text {th }}$ and $m^{\text {th }}$ harmonic number, they are not exponents.

The minimization algorithm can be generalized and easily extrapolated to a higher number of components. As an example for the case for 3 components we would write equations (Eq 6) and (Eq 7) for three harmonics, obtaining 6 equations and 9 unknowns. Using (Eq 8) we would substitute all $f_{1}$ in terms of $f_{2}$ and $f_{3}$, using (Eq 5 ) we would substitute all $s_{i}$ coordinates in terms of the $g_{i}$ coordinates, and using (Eq 9) we would substitute all higher harmonics in terms of the first harmonic. This would lead to a set of 6 equations and 5 unknowns. In each of these equations, all the terms can be sent to one side of the equality, having a set of 6 equalities to zero. The squared sum of the non-zero sides is the quantity that the minimization algorithm has to minimize. Since this quantity is a sum of squares, in the following section we call it $\chi^{2}$ although it is not a sum of normal functions. 
Figure 1 in the main text of the paper shows the graphical representation of the phasor plot for two single exponential lifetimes and their $2^{\text {nd }}$ harmonics both on the universal circle. The linear combination of the two single exponential components with phasors for the fundamental and the harmonics maps to a point that lies on the line that joins the two positions on the universal circle (figure 1A). This figure shows the use of the second harmonic to resolve the system made of two single exponential components.

For 3 components the reasoning is similar but we need an additional constraint that the combination of each lifetime and the harmonics must give the same fractional intensities for all 3 components as shown in figure 2 and analytically in Eq 6 and 7.

We note here that the origin of the fitting problem is in Eq 6 and 7. The data to be fit are $G^{(h)}$ and $S^{(h)}$ which are the sum of linear combinations of the $g_{i}^{(h)}$ and $s_{i}^{(h)}$ weighted by the intensity fractions $f_{i}$, so that the same value for the right hand side (the measured data points) can be obtained by many combinations of lifetimes and intensity fractions. In other words the values of the lifetimes and the intensity fraction are constrained by a linear combination rather than being orthogonal. As a consequence, any small error in the harmonics propagates to all the parameters (lifetime and intensity fraction) increasing the uncertainty of the solution. This is the origin of the relatively large value of the errors in the fit. The more components we have, to larger is the errors and we needed in the fits to fix at least one of the components to determine the other. In the fitting of equations are Eq 6 and 7, which are bilinear, the knowledge of one lifetime or intensity fractional component is sufficient to solve for the other parameters. The consequence of the structure of the equations is that we cannot continue to add component unless the harmonics are known with very small errors. A corollary of this reasoning as that we have to be aware that the transform of data from the time domain due to the distortion caused by the histogram of delay time is not good enough to obtain the exact values of the harmonics. Using the simulation program (available for free download at https://www.Ifd.uci.edu/) we can determine to amount of noise that can be tolerated for the blind determination of lifetimes and fractional intensity as it is done in the main part of this paper. A look at figure $2 \mathrm{~B}$ shows that the harmonic (the open circles) for some combinations of lifetimes and fractions tend to be aligned, which further complicates the fit. 


\section{Example Application and analysis pipeline: Dyes in live cells.}

Here we present a pipeline for analysis of live cells using our method, which is the one we followed for the data we displayed in figure7 in the main body of the paper (cells with Acridine Orange, Nuc Blue and Rose Bengal). Figure S1 shows each of the three dyes individually in cells, having been imaged in our FLIM microscope. From this data we measured the lifetimes of the pure components which were then used in the paper to compare with the recovered components in the case of the cells with the three dyes together. Note that Acridine Orange and Nuc Blue have relatively close lifetime and as we show in our simulations (figure 4 of the manuscript) they are harder to resolve independently. The three dyes do not appear on the universal circle due to the fact that dyes inside the cells often deviate from their monoexponential behavior in solution and show complicated behavior, often due to differences in binding sites. Furthermore, each pixel contains other sources of light, autofluorescence, which in general tends to pull the phasor data towards the center of the plot due to it being a combination of many lifetimes.

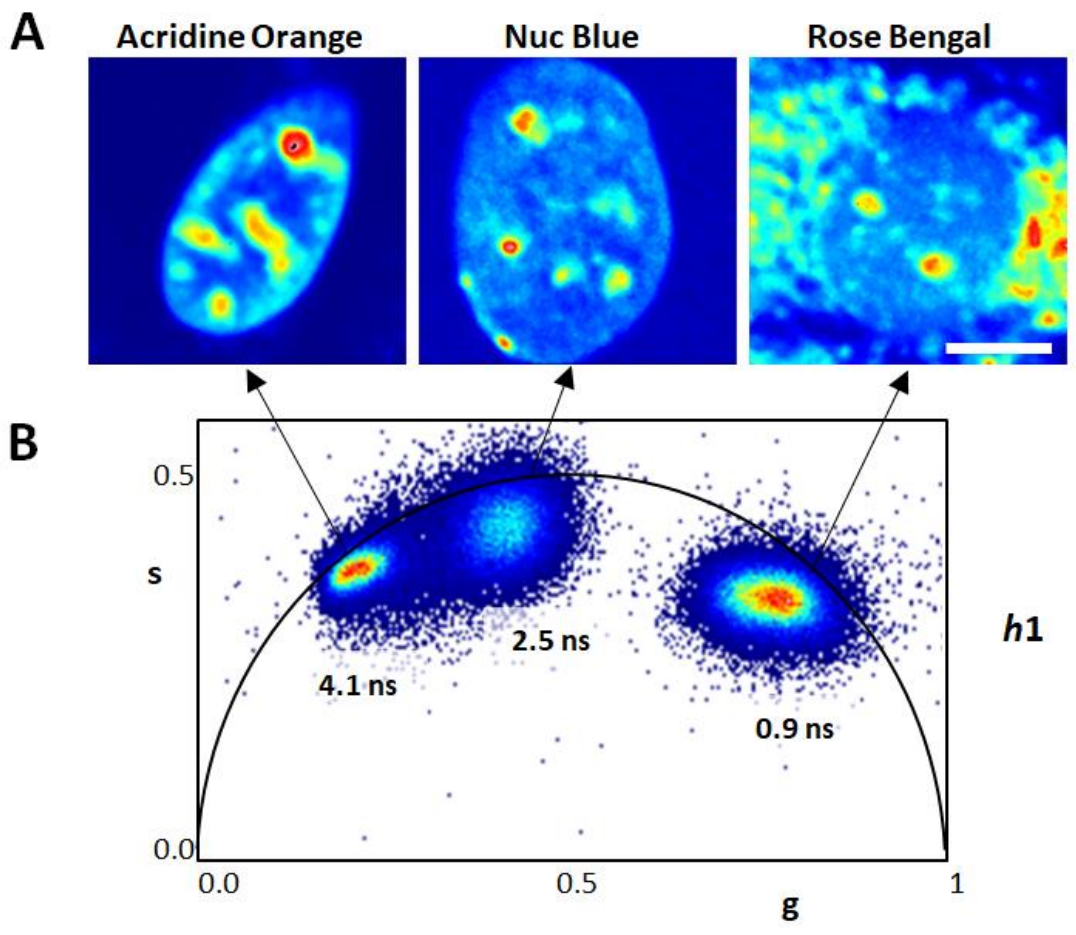

Figure S1. Lifetime and position on the phasor plot of the individual dyes. A) FLIM images of cells stained individually with Acridine Orange, Nuc Blue or Rose Bengal. Scale bar $5 \mu \mathrm{m}$. B) Phasor position for each of the dyes in the first harmonic (h1) at $80 \mathrm{MHz}$, and the corresponding lifetime of the center of the distribution.

In the minimization step, we use as a convergence criterion the value of the surface at the solution. We name it $\chi^{2}$ because it is a squared sum of differences of terms (normalized to the number of terms). We found a lower value of this $\chi^{2}$ when fitting the data to three components than for two and for four and for this reason we show the analysis for three components. Figure S2 shows a schematic of the data analysis pipeline which is also described below. 


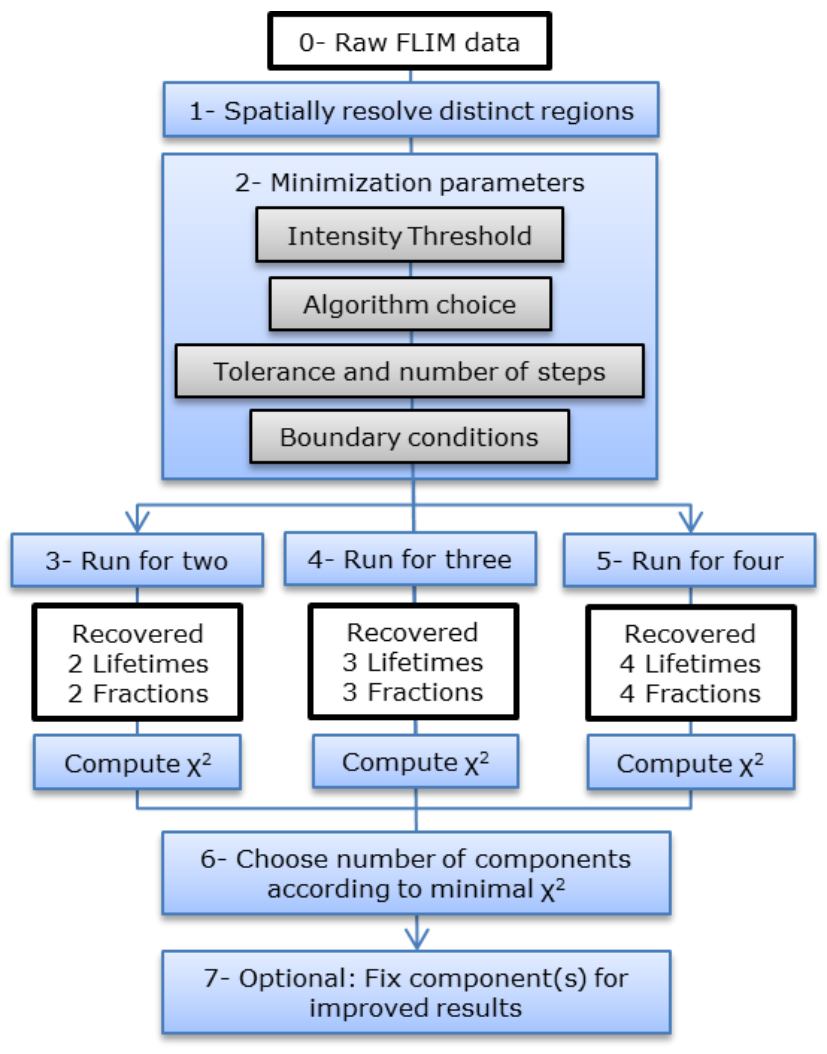

Figure S2. Workflow for data analysis of the blind component recovery.

1. Identify distinct populations on the phasor plot in order to spatially separate regions where to run the blind analysis.

2. General parameters to perform blind components analysis.

2.1. Discard pixels with less than a threshold count (for figure7 in the paper we used 40counts).

2.2. Choose minimization method (Levenberg-Marquardt, Nelder-Mead Simplex, SQP, alternating versions etc.).

2.3. Set method tolerance and number of steps for minimization.

2.4. Set number of iterations in each pixel

3. Set the number of components for the analysis.

4. Run the algorithm for different number of components and obtain a goodness of fit criteria like the $\chi^{2}$.

5. Determine the minimum number of components needed for a given sample using the mentioned criteria

6. Optionally fix some components if additional data is available. 